\title{
Systematic study of structures and stabilities of fullerenes
}

\author{
B. L. Zhang, C. H. Xu, C. Z. Wang, C. T. Chan, and K. M. Ho \\ Ames Laboratory, and Department of Physics and Astronomy, Iowa State University, Ames, Iowa 50011
}

(Received 14 January 1992)

\begin{abstract}
We describe a scheme to generate the ground-state network for carbon clusters. Combining this scheme with an accurate tight-binding potential for carbon, we have systematically studied the ground-state structures of fullerenes from $\mathrm{C}_{20}$ to $\mathrm{C}_{94}$. Clusters of sizes 60,70 , and 84 are found to be energetically more stable than their neighbors. The occurrence of the more abundant fullerene sizes is related to the fragmentation stability and chemical reactivity of the clusters obtained from our calculations.
\end{abstract}

With the breakthroughs ${ }^{1}$ in synthesizing macroscopic quantities of $\mathrm{C}_{60}$, experimentalists ${ }^{2}$ have unambiguously characterized its highly symmetric icosahedral structure, confirming the theoretical prediction made several years ago by Kroto et al. ${ }^{3}$ Now that many more large carbon fullerenes are being isolated, ${ }^{4-6}$ determining their structures becomes a big challenge to both experimentalists and theorists.

One important piece of experimental evidence of the cluster structure comes from measurement of the ${ }^{13} \mathrm{C} \mathrm{nu}$ clear magnetic resonance (NMR) spectra. From the number of NMR lines, it is possible in some cases to determine the symmetry of the molecule. ${ }^{4}$ However, it is hard to pinpoint the symmetry exactly when the structure has a low symmetry. Even when the symmetry is known, the structure is still undetermined because large fullerenes can have more than one structure under the same symmetry.

On the theoretical side, existing approaches can be divided into two categories. The first one is to construct several plausible networks from geometry and symmetry considerations, ${ }^{7-13}$ and then pick out the best one after calculating the total energy or the energy separations between the highest-occupied molecular-orbital (HOMO) and the lowest-unoccupied molecular-orbital (LUMO) of each candidate. Approaches based on symmetry consideration $^{7-9}$ are useful only for clusters of certain special sizes and the deduced structures usually possess high point-group symmetry. But the recently isolated ${ }^{4} C_{76}$ has the relatively low $\mathrm{D}_{2}$ symmetry. To overcome this limitation, Manolopoulos introduced a new geometry method ${ }^{10}$ which uses the computer to search for every possible network. Since there is no symmetry constraint, the number of possible isomers is huge. For example, there are 1812 distinct isomers for $\mathrm{C}_{60}$ alone. ${ }^{14}$ Although the isolatedpentagon rule can be used to reduce the possible structures to a limited number, the number of isolatedpentagon structures grows very fast as the clusters get bigger and it is quite expensive to test each one of them. Moreover, with the exception of $\mathrm{C}_{60}$ and $\mathrm{C}_{70}$, fullerenes with sizes less than 70 have no isolated-pentagon isomers. It is not efficient to test thousands of possible isomers to pick out the ground-state isomer.

In the second approach, ${ }^{15-17}$ the ground-state structures are obtained by simulated annealing. This approach requires an accurate and efficient interatomic potential. It is still beyond our present computer capability to perform first-principles molecular dynamics for large fullerenes over tens of thousands steps. A more serious problem is that the system will often be trapped in some metastable high-energy structure because the strong directional bonding of carbon creates large energy barriers between various metastable structures. For example, in the work of Ballone and Milani, ${ }^{15}$ it is reported that structures for $\mathrm{C}_{62}$ and $\mathrm{C}_{64}$ always contain seven-member ring defects although we now know that a much more stable structure exists with only five- and six-membered rings. The energy to break the carbon bonds and rearrange the network is so high that it is difficult to reach the ground state unless one starts from a structure which already has the correct topology.

To overcome the difficulties mentioned above, we devised a scheme for generating energetically favorable structures for large fullerenes. In this scheme, we first focus on generating the correct topological networks for energetically favorable structures. Instead of looking at the network connecting individual atoms, we focus our attention on the "face-dual" network obtained by linking the centers of each of the polygonal face of the cage structure. Since each atom of the fullerene is threefold coordinated, the face-dual network consists of a triangular mesh. For a fullerene with $N$ atoms, the number of polygonal faces is $N / 2+2 .^{7}$ We represent each of the faces by a point lying on a predetermined surface (a sphere for example) and obtain the ground-state network by simulated annealing. Initially these points are placed at random and interact with one another through a two-body repulsive potential. The face-dual network obtained can then be inverted to obtain the fullerene structure. In this scheme, whether a face is pentagonal or hexagonal is determined by the number of nearest-neighboring faces. Thus the change from pentagon to hexagon can occur easily with a small energy barrier and even for quite large number of atoms, the system does not get trapped in local metastable states. Also, this scheme distributes the faces as evenly as possible on the constrained surface and the resultant topological networks tend to separate the pentagonal faces as far apart as possible which makes the structures generated good candidates for low-energy fullerene structures. By varying the shape of the constraining surface (changing 
the sphere to ellipsoids), we can obtain a set of energetically favorable topological networks. The ground-state structure is then found by unconstrained simulated annealing of the atomic arrangements for each of the candidate network using an accurate tight-binding moleculardynamics scheme and comparing the energies of the various competing isomers.

The tight-binding potential model we used has the total-energy expression:

$$
E\left(\left\{\mathbf{r}_{i}\right\}\right)=\sum_{n}^{\text {occupied }}\left\langle\psi_{n}\left|H_{\mathrm{TB}}\left(\left\{\mathbf{r}_{i}\right\}\right)\right| \psi_{n}\right\rangle+E_{\text {rep }}\left(\left\{\mathbf{r}_{i}\right\}\right) .
$$

The first term in (1) is the electronic band-structure energy calculated by a parametrized tight-binding Hamiltonian $H_{\mathrm{TB}}\left(\left\{\mathbf{r}_{i}\right\}\right)$, and the second term is a short-ranged repulsive energy. The orthogonal $s p^{3}$ basis tight-binding Hamiltonian $H_{\mathrm{TB}}\left(\left\{\mathbf{r}_{i}\right\}\right)$ is described by the following parameters: $\varepsilon_{s}$ and $\varepsilon_{p}$ are on-site atomic energies, $v_{s s \sigma}\left(r_{i, j}\right)$, $v_{s p \sigma}\left(r_{i, j}\right), v_{p p \sigma}\left(r_{i, j}\right)$, and $v_{p p \pi}\left(r_{i, j}\right)$ are overlap parameters as function of interatomic distance $r_{i, j}$. The repulsive energy is in the form of $E_{\text {rep }}=\Sigma_{i} f\left[\Sigma_{j} \phi\left(r_{i, j}\right)\right]$. The above parameters and functions were fitted to first-principles calculation results of electronic band structure and volume-dependent binding energies of various crystalline carbon phases. The optimized parameters not only reproduce the binding energies and bond lengths of crystalline carbon with different coordination numbers, but also describe well the properties of the liquid and amorphous states. ${ }^{18}$ The speed of this calculation is hundreds of times faster than regular first-principles molecular dynamics, so we can easily handle systems with several hundred atoms. The reliability of the TB potential for clusters is demonstrated by accurate ground-state geometries of small clusters in comparison with $a b$ initio quantum chemical calculations. ${ }^{19}$ In the range $5 \leq N \leq 11$, we found that odd-numbered clusters prefer a linear structure, while even-numbered clusters prefer a ring structure. These results cannot be obtained by classical potentials which neglect quantum mechanical effects. Since the potential parameters were fitted to bulk properties, we expect that our results for large clusters should be even more reliable.

With the efficient face-dual network generation method and the accurate tight-binding molecular-dynamics scheme described above, we have systematically studied every even-numbered carbon cluster ranging from $\mathrm{C}_{20}$ to $\mathrm{C}_{94}$. Except in the case of $\mathrm{C}_{22},{ }^{20}$ all the lowest-energy cages obtained by the present scheme contain only fiveand six-membered rings. The number of five-membered rings is exactly 12 in each fullerene, consistent with the well-known Euler's theorem. In the range of $N=20$ to 70 , the $I_{h}$ isomer of $\mathrm{C}_{60}$ and the $\mathrm{D}_{5 h}$ isomer of $\mathrm{C}_{70}$ are the only two isomers having no adjacent pentagons. For $72 \leq n \leq 94$, all ground-state structures are found to satisfy the isolated-pentagon rule. These results demonstrate that the present scheme is indeed better than earlier simulated annealing methods ${ }^{15-17}$ which deals with the atomic network directly. More details of the structural properties are published elsewhere. ${ }^{21}$ The present scheme reproduces well the ground-state structures of all the experimentally determined fullerenes, i.e., the $I_{h}$ structure of $C_{60}$, the $D_{5 h}$ structure of $C_{70}$, and even the chiral $\left(D_{2}\right)$ structure of $\mathrm{C}_{76 .}{ }^{4}$ Moreover, the present tight-binding calculation on $\mathrm{C}_{60}$ yields a heat of formation of $0.40 \mathrm{eV}$ per atom (with respect to that of bulk graphite) and a HOMO-LUMO energy gap of $1.61 \mathrm{eV}$. These values agree quite well with the $a b$ initio local-density approximation calculation results of 0.40 and $1.71 \mathrm{eV}$, respectively. 22

The heats of formation of the ground-state cages as a function of cluster size obtained by our present scheme are plotted in Fig. 1. We found that the formation energies of the small cages grow rapidly as the cluster size is increased. This behavior can be related to the rapidly decreasing number of adjacent pentagons as the cluster size increases. On the other hand, the energies of large fullerenes which have no adjacent pentagons are found to increase at a slower rate. The most interesting feature is that $\mathrm{C}_{60}, \mathrm{C}_{70}$, and $\mathrm{C}_{84}$, which are the magic numbers observed in experiments, are found to be more stable than their neighbors.

Although the extraordinary abundance of $\mathrm{C}_{60}$ and $\mathrm{C}_{70}$ has been known for several years, the underlying origin of the phenomena is still not well understood. Recently, abundance peaks have also been observed ${ }^{6}$ for $C_{76}, C_{78}$, $\mathrm{C}_{84}, \mathrm{C}_{90}$, and $\mathrm{C}_{94}$, but not for every even-numbered clusters in this range. It seems that a complete explanation of the experimental observations has to involve kinetic factors in addition to thermodynamic stability. Otherwise all fullerenes larger than $C_{70}$ would be more abundant than $\mathrm{C}_{60}$ according to their cohesive energies. From our present systematic study, we found that the abundance peaks of the mass spectra can be correlated with the fragmentation stabilities and electronic HOMO-LUMO energy separations of the fullerenes.

Since the exact fragmentation process of carbon fullerenes is still unclear at present, we limit our consideration to a process with successive $C_{2}$ loss as suggested by O'Brien et al. ${ }^{23}$ The fragmentation energy in this process is defined as

$$
E_{\text {frag }}(N)=E_{\text {coh }}(N)-E_{\text {coh }}(N-2)-E_{\text {coh }}(2) .
$$

Here the $E_{\text {coh }}(N)$ is the cohesive energy of $\mathrm{C}_{N}$ and $E_{\text {frag }}(N)$ is the energy needed for $\mathrm{C}_{N}$ to fragment into

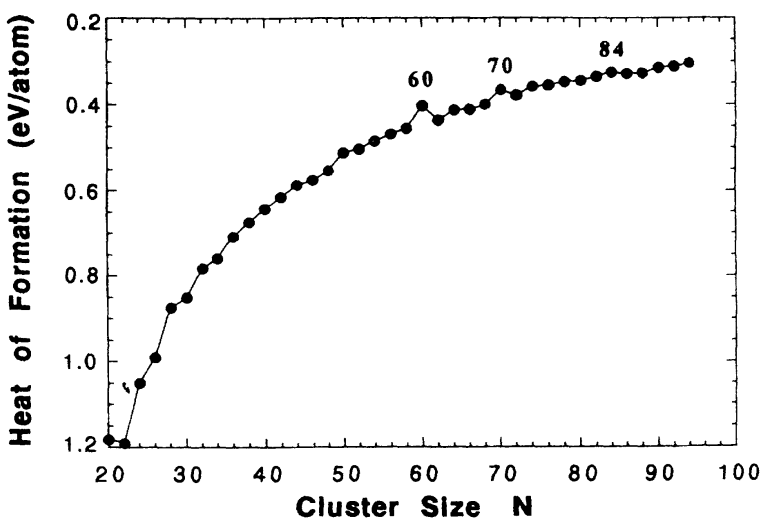

FIG. 1. The heat of formation of carbon clusters relative to bulk graphite as a function of cluster size $N$. 
$\mathrm{C}_{N-2}$ and $\mathrm{C}_{2}$. Therefore clusters with larger fragmentation energy will be more stable against fragmentations. In Fig. 2(a), the fragmentation energy as a function of cluster size obtained from our present study are plotted. It is clearly shown that $\mathrm{C}_{60}$ and $\mathrm{C}_{70}$ have very large fragmentation energies with respect to their neighboring fullerenes. These results may explain why these two fullerenes are superstable. However, it is not clear from the fragmentation energy that in the range of $N=72$ to 94 , why $\mathrm{C}_{74}, \mathrm{C}_{80}$, $\mathrm{C}_{86}$, and $\mathrm{C}_{88}$ do not show abundant peak in the mass spectra.

Recently, Manolopoulos ${ }^{10}$ suggested using the HOMOLUMO energy separation as a criterion for the kinetic stability of fullerenes. While it is still not clear to us how the HOMO-LUMO gap can control fullerene formation at high temperature, we believe that it is a key parameter controlling the chemical reactivity of the fullerene during the extraction and isolation process. A big HOMOLUMO energy separation makes it more difficult to extract electrons from the low-lying HOMO or add electrons to the high-lying LUMO. It is easier for the clusters with small HOMO-LUMO energy gap to react with the solvent and other encountered chemicals. A bigger HOMO-LUMO gap might also make the cluster more stable towards further accretion of extra $\mathrm{C}$ atoms. In Fig. 2(b), our result of the HOMO-LUMO gap is plotted as a function of cluster size. It is clearly shown that the $\mathrm{C}_{60}$ and $C_{70}$ not only have very large fragmentation energies, but also have very large HOMO-LUMO gaps. These results are strongly correlated to the extraordinary abundance of these two fullerenes. It is also interesting to see that $\mathrm{C}_{74}, \mathrm{C}_{80}, \mathrm{C}_{86}$, and $\mathrm{C}_{88}$, which are absent in the mass spectra, all have very small HOMO-LUMO gaps. Due to the chemical reactivity discussed above, these fullerenes should be less stable during the isolation process.

Finally, we would like to point out that an explanation of experimental mass spectra based solely on the consideration HOMO-LUMO energy separations would not be satisfactory. An obvious example is $C_{72}$ which has very large HOMO-LUMO energy separation but it is not abundant in the mass spectra because its fragmentation energy strongly favors losing a dimer to become $\mathrm{C}_{70}$. Our present study shows that in order to explain the mass spectra of larger fullerenes, it is necessary to consider both the HOMO-LUMO gaps and fragmentation energies simultaneously. The HOMO-LUMO gap measures the stabili-

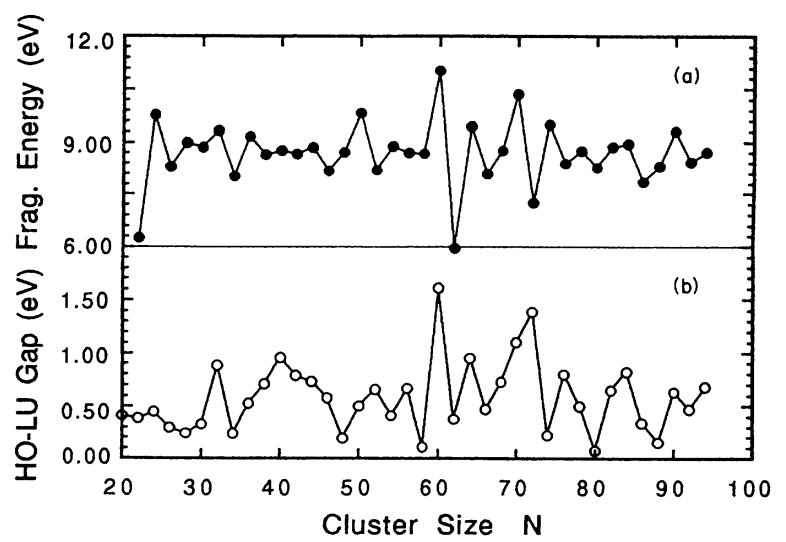

FIG. 2. (a) The fragmentation energy and (b) HOMOLUMO energy separation as a function of cluster size.

ty of the cluster against further chemical reaction, while the fragmentation energy measures the stability of the clusters against further fragmentation. The only exception in our calculation is $\mathrm{C}_{64}$. It has quite high peaks in both stabilities, but it is not observed in experiments. We suspect that $\mathrm{C}_{64}$ is too close to the superstable $\mathrm{C}_{60}$ and it may be unstable towards a fragmentation with a loss of $\mathrm{C}_{4}$.

In summary, we have shown that the new cage network generation scheme can efficiently and accurately predict the network of large fullerenes. The ground-state structures and energies obtained from tight-binding molecular dynamics agree very well with the existing experimental results. From this systematic study, we found that the superstable carbon clusters not only have high fragmentation stability but also have a large HOMO-LUMO energy separation. On the contrary, the fullerenes which are hard to obtain in experiments have a tendency towards fragmentation or further chemical reactions.

Ames Laboratory is operated for the U.S. Department of Energy by Iowa State University under Contract No. W-7405-Eng-82. This work was supported by the Director for Energy Research, Office of Basic Energy Sciences, including a grant of computer time at the National Energy Research Supercomputing Center at Livermore and by NSF Grant No. DMR-8819379.
'W. Krätschmer, L. D. Lamb, K. Fostiropoulos, and D. R. Huffman, Nature (London) 347, 354 (1990).

${ }^{2}$ R. Taylor, J. R. Hare, A. K. Abdule-Sada, and H. J. Kroto, J. Chem. Soc. Chem. Commun. 20, 1423 (1990).

${ }^{3}$ H. W. Kroto, J. R. Heath, S. C. O'Brien, R. F. Curl, and R. E. Smalley, Nature (London) 318, 162 (1985).

${ }^{4}$ R. Ettl, I. Chao, F. Diederich, and R. L. Whetten, Nature (London) 353, 149 (1991).

${ }^{5}$ K. Kikuchi, N. Nakahara, M. Honda, S. Suzuki, K. Saito, H. Shiromaru, K. Yamauchi, I. Ikemoto, T. Kuramochi, S. Hino, and Y. Achiba, Chem. Lett. 1991, 1607.

${ }^{6}$ F. Diederich, R. Ettl, Y. Rubin, R. L. Whetten, R. Beck, M.
Alvarez, S. Anz, D. Sensharma, F. Wudl, K. C. Khemani, and A. Koch, Science 252, 548 (1991).

7P. W. Fowler, J. E. Cremona, and J. I. Steer, Theor. Chim. Acta 73, 1 (1988).

${ }^{8}$ T. G. Schmalz, W. A. Seitz, D. J. Klein, and G. E. Hite, J. Am. Chem. Soc. 110, 1113 (1988).

${ }^{9}$ D. Bakowies and W. Thiel, J. Am. Chem. Soc. 113, 3704 (1991).

${ }^{10}$ D. E. Manolopoulos, J. C. May, and S. E. Down, Chem. Phys. Lett. 181, 105 (1991).

${ }^{11}$ P. W. Fowler, J. Chem. Soc. Faraday Commun. 87, 1945 (1991) 
${ }^{12}$ D. E. Manolopoulos, J. Chem. Soc. Faraday Commun. 87, 2861 (1991).

${ }^{13}$ P. W. Fowler, R. C. Batten, and D. E. Manolopoulos, J. Chem. Soc. Faraday Commun. 87, 3103 (1991).

${ }^{14}$ D. E. Manolopoulos and P. W. Fowler, J. Chem. Phys. 96, 7603 (1992).

${ }^{15}$ P. Ballone and P. Milani, Phys. Rev. B 42, 3201 (1990).

16 J. R. Chelikowsky, Phys. Rev. Lett. 67, 2970 (1991).

${ }^{17}$ C. Z. Wang, C. H. Xu, C. T. Chan, and K. M. Ho, J. Phys. Chem. 96, 3563 (1992).

${ }^{18}$ For details of the tight-binding model, see C. H. Xu, C. Z.
Wang, C. T. Chan, and K. M. Ho, J. Phys. Condens. Matter 4, 6047 (1992).

${ }^{19}$ K. Raghavachari and J. S. Binkley, J. Chem. Phys. 87, 2191 (1987).

${ }^{20} \mathrm{C}_{22}$ cannot form a cage with five- and six-member rings only; please see Ref. 7 for more detailed discussion.

${ }^{21}$ B. L. Zhang, C. Z. Wang, and K. M. Ho, Chem. Phys. Lett. 193, 225 (1992).

22 J. L. Martins (private communication).

${ }^{23}$ S. C. O'Brien, J. R. Heath, R. F. Curl, and R. E. Smalley, J. Chem. Phys. 88, 220 (1988). 